\title{
Chemical and strain effects on Boron-doped $\mathrm{Si}(100)$
}

\author{
M. Ramamoorthy, E. L. Briggs, and J. Bernholc \\ Department of Physics, North Carolina State University, Raleigh, North Carolina 27695-8202
}

(Received 22 September 1998)

\begin{abstract}
Theoretical investigations uncover complex strain-induced modes of boron ordering at the $\operatorname{Si}(100)$ surface, which are highly anomalous in comparison with other group III impurities. The fundamental units of the clustering process are subsurface pairs of B atoms. The structural relaxations around segregated B impurities are substantial and the induced strain fields couple with the $(2 \times 1)$ dimer reconstruction of the surface to stabilize complex, zigzag modes of ordering. Impurity configurations exist that are strongly bound with respect to isolated subsurface impurity pairs up to the critical doping level of 0.5 monolayer. Above this doping level, however, all modes of ordering of the impurities at the surface are repulsive. A number of experimental observations are explained and interesting structures are predicted. [S0163-1829(99)07207-0]
\end{abstract}

\section{INTRODUCTION}

There is an increasing trend towards miniaturization in the manufacture of semiconductor devices. For submicron devices, intense effort has been directed towards producing ordered, heavily doped structures with extremely narrow impurity distribution profiles. ${ }^{1}$ An example of such a structure is a delta layer, where the impurities are localized in a nearly two-dimensional region. In silicon, $p$-type delta layers have been developed recently, by incorporation of high levels of boron impurities into the $\mathrm{Si}(100)$ surface. This has stimulated a great deal of interest in the properties of heavily B-doped $\mathrm{Si}(100)$, revealing a system with extremely complex structural and electronic properties. ${ }^{2-6}$

The electrical carrier density of these heavily doped structures increases linearly as a function of impurity concentration, up to a critical doping level corresponding to half a monolayer (ML) of B impurities. ${ }^{3}$ At precisely $0.5 \mathrm{ML}$ doping, low-energy electron diffraction (LEED) reveals a B-induced reconstruction, with $(2 \times 1)$ periodicity like the clean surface, but with a number of distinct features. All the incorporated impurities are found to be fully activated. Above this doping level, the carrier density is found to saturate and the LEED background increases sharply, implying that additional impurities do not order. When the B-doped surfaces are capped with epitaxial Si layers at low temperatures, there is minimal impurity disordering. Grazing angle $\mathrm{x}$-ray-diffraction studies of such structures reveal that the impurity arrangement is indeed nearly two dimensional, with any disordering being limited to at most a few atomic monolayers. ${ }^{4}$

Detailed structural information on B-doped $\mathrm{Si}(100)$ has been obtained using scanning tunneling microscopy (STM). One surprising feature of the published observations are the extent to which they vary, depending on the methods of surface processing. Wang, Hamers, and Kaxiras, ${ }^{7}$ who investigated B-doped $\mathrm{Si}(100)$ surfaces obtained by thermal decomposition of adsorbed diborane, observed islands with highconcentration levels of boron and $c(4 \times 4)$ and $(4 \times 4)$ reconstructions. The surface electronic density of states and chemical reactivity on these heavily doped surfaces were shown to exhibit significant lateral variation parallel to the surface. This raised the intriguing possibility of engineering nanoscale variations in surface properties via control of the ordering of B-induced surface reconstructions. However, other investigators, ${ }^{8,9}$ who induced boron segregation to the surface via high-temperature annealing, did not observe such reconstructions. Furthermore, it was suggested by one group of investigators ${ }^{8}$ that the observed $c(4 \times 4)$ and $(4 \times 4)$ reconstructions are likely to be stabilized by hydrogen impurities released during thermal decomposition of diborane.

Several distinct models of boron ordering in the adlayer and in subsurface layers have been proposed and no consensus has been reached so far. ${ }^{6-12}$ This is in contrast to the progress that has been made in characterizing the segregation and ordering of a number of other electrically active impurities at $\mathrm{Si}(100)$, where impurity segregation leads to saturation of surface dangling bonds. Indeed, at a critical doping level, the surface becomes fully passivated. For group V donors like $\mathrm{P}, \mathrm{As}$, and $\mathrm{Sb}$, this occurs at a doping level of one monolayer $(\mathrm{ML})$, where a $(2 \times 1)$ reconstruction made up of a monolayer of impurity dimers covers the surface. ${ }^{13-15} \mathrm{On}$ the other hand, for group III acceptors like $\mathrm{Al}, \mathrm{Ga}$, and In, the critical doping level corresponds to $0.5 \mathrm{ML}$, where impurity dimers order in the adlayer, forming a $(2 \times 2)$ reconstruction. ${ }^{16,17}$ In comparison, the properties of B-doped $\mathrm{Si}(100)$ are anomalous. The surface with $0.5 \mathrm{ML}$ of boron, which is the maximum doping level at which impurity ordering is observed, has a $(2 \times 1)$ reconstruction and an appreciable concentration of dangling bonds. 3,6

In this paper, we present a systematic description of boron segregation and ordering at the $\mathrm{Si}(100)$ surface, from low to high doping levels, using the results of first-principles totalenergy calculations. We find that substitutional boron impurities prefer to order in the second layer of the surface. Extensive strain results from boron substitution, favoring complex ordering modes with zigzag orientation with respect to the surface Si dimer rows. A doping level of 0.5 ML of impurities indeed corresponds to a critical threshold. At and below this doping level, impurity clustering in the nearsurface region is energetically favorable, while impurity aggregation becomes increasingly repulsive with increasing doping above this level. The lowest energy structure at 0.5 ML doping has $c(4 \times 4)$ periodicity, which is in accord with 
experiment. ${ }^{7}$ A number of low-energy metastable impurity configurations are uncovered, which favors the formation of complex domain structures.

\section{CALCUlations}

The total energies of structures were calculated using the density-functional method, ${ }^{18}$ with the local-density approximation for the exchange and correlation contribution. Ions were represented using norm conserving pseudopotentials ${ }^{19}$ and electronic wave functions were calculated using a realspace multigrid-based approach. ${ }^{20}$ Calculations of the bulk Si lattice constant and the structure of the $(2 \times 1) \mathrm{Si}(100)$ surface gave results in very good agreement with experiment and with earlier theoretical work. Impurities on $(2 \times 1)$ $\mathrm{Si}(100)$ were studied using $(4 \times 4)$ surface supercells. Initial calculations were done with six-layered slabs, the $\Gamma k$ point, a vacuum layer corresponding to six Si layers and a real-space grid spacing of 0.4 a.u. (corresponding to a plane-wave cutoff of $31 \mathrm{Ry}$ ). Important quantities were refined by carrying out calculations with ten-layer slabs, a vacuum layer corresponding to ten Si layers, the $(0.25,0.25,0)$ special $k$-point and a real-space grid spacing of 0.3 a.u.

\section{SURFACE SEGREGATION AT LIGHT DOPING LEVELS}

We first report results on the segregation of isolated substitutional boron impurities at or near the $(2 \times 1) \mathrm{Si}(100)$ surface, to lay the foundations for the subsequent discussions of boron ordering at high doping levels. This surface has a complex corrugated structure, with the surface $\mathrm{Si}$ atoms ordered in rows of dimers, separated by wide trenches. ${ }^{21-23}$ Each surface atom is only threefold coordinated, unlike the fourfold coordination found in bulk $\mathrm{Si}$, which results in a high density of dangling bonds at the surface. A number of common donor and acceptor impurities in Si segregate to this surface, as they prefer threefold coordination. Group V donors like $\mathrm{P}, \mathrm{As}$, and $\mathrm{Sb}$ prefer dimer configurations in the top layer of the surface, while group III acceptors like $\mathrm{Al}, \mathrm{Ga}$, and In are observed to dimerize and order in the adlayer. ${ }^{13-17}$

In Fig. 1, we present the energy of an isolated substitutional B impurity as a function of distance from the surface. The lowest energy configuration is found to be in either the first or the second layer of the surface, while those in deeper subsurface layers are significantly higher in energy. This is a very surprising result as the chemical coordination of the impurity in the two layers is completely different. Furthermore, it is highly anomalous in comparison with the behavior of the other dopants mentioned above.

One feature common to all substitutional boron configurations is the large relaxations of its Si neighbors, due to the small atomic size of B relative to $\mathrm{Si}$. In the top layer impurity configuration, the surface $\mathrm{Si}-\mathrm{B}$ heterodimer is flat and lowered by almost $0.5 \AA$ compared to the average height of the tilted surface Si dimers. This is a very large displacement, almost $40 \%$ of the interlayer spacing. For the second layer impurity configuration, the surface $\mathrm{Si}$ dimers bonded to the impurity experience downward relaxations of a roughly similar magnitude. These large relaxations indicate that strain effects are very important in B segregation and would be

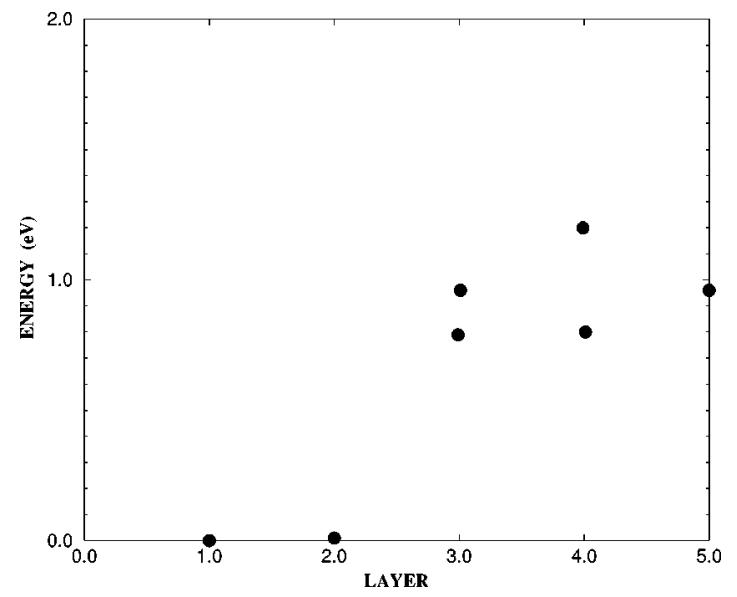

FIG. 1. The energy of a substitutional B impurity as a function of depth below the surface. There are two different types of sites in layers three and four, one of which is below the dimer rows and the other beneath the trenches between dimer rows. The energy of the substitutional impurity in the bulk, with reference to the impurity in the surface layer, is $+1.6 \mathrm{eV}$.

expected to play an increasingly important role as the doping level is increased.

Strain effects were shown to be predominant in the segregation and ordering of carbon impurities at the $\mathrm{Si}(100)$ surface. ${ }^{24,25}$ Carbon is a first row impurity like $\mathrm{B}$, but it is isovalent with $\mathrm{Si}$. If strain dominated over electronic effects, the segregation of B impurities should be qualitatively similar to that of C. However, the results shown in Fig. 1 for B are very different from the corresponding ones for $\mathrm{C}$ reported by Tersoff, ${ }^{24}$ who used empirical potentials. First, Tersoff found that the energy of the $\mathrm{C}$ impurity in the second layer is comparable (within $0.1-0.2 \mathrm{eV}$ ) to that of the lowest energy configuration in layers three and four. In contrast, our results indicate that the corresponding energy difference for $\mathrm{B}$ is much larger (over $0.7 \mathrm{eV}$ ).

Turning to deeper layers, there are two distinct types of sites in the third and the fourth layers of the surface. Following Tersoff, we label the sites underneath a surface dimer row as $\alpha$ and those underneath the trenches between surface dimer rows as $\beta$. The former (latter) sites are known to be under compressive (tensile) stress. Tersoff found that $\mathrm{C}$, being a small impurity, strongly preferred (by over $1.0 \mathrm{eV}$ ) an $\alpha$ site over a $\beta$ site. Again, this is not the case with boron. We find that a B impurity in the third layer prefers the $\beta$ site by $0.3 \mathrm{eV}$ over the $\alpha$ site. In the fourth layer, the ordering is reversed. Now, the $\beta$ site is higher in energy over the $\alpha$ site, by about $0.4 \mathrm{eV}$. In summary, the segregation of isolated $\mathrm{B}$ impurities to $\mathrm{Si}(100)$ is unique, compared to other common impurities in Si. The process is influenced by both electronic and strain effects and neither can be considered to be predominant.

\section{PAIRING OF SEGREGATED BORON IMPURITIES}

As the concentration of segregated impurities increases, there is a significant probability of two of them being in proximity to each other. To quantify the nature of interaction between substitutional impurities, we calculated the energies of a number of configurations where pairs of impurities are 
TABLE I. The energies of pairs of substitutional boron impurities near the $\mathrm{Si}(100)$ surface, using the notation established in Fig. 2 for labeling impurity configurations.

\begin{tabular}{cc}
\hline \hline Impurity pair & Binding energy \\
\hline A1-A2 & +0.4 \\
A1-A3 & +0.3 \\
B1-B2 & 0.0 \\
B1-B3 & 0.0 \\
B2-B3 & +0.2 \\
B2-B4 & +0.2 \\
B3-B4 & 0.0 \\
C1-C2 & +1.2 \\
C1-C3 & +1.3 \\
\hline \hline
\end{tabular}

nearest neighbors, next-nearest neighbors and even thirdnearest neighbors. The salient results are presented in Table I, using the notation established in Fig. 2 to label the various structures. The geometry of the $\operatorname{Si}(100)$ surface is such that apart from the first layer, impurities within a given surface layer can be no closer than next-nearest neighbors. Obviously, there are configurations where impurity pairs may be nearest neighbors, namely when the two impurities are in adjacent layers. Without exception, all nearest-neighbor con-

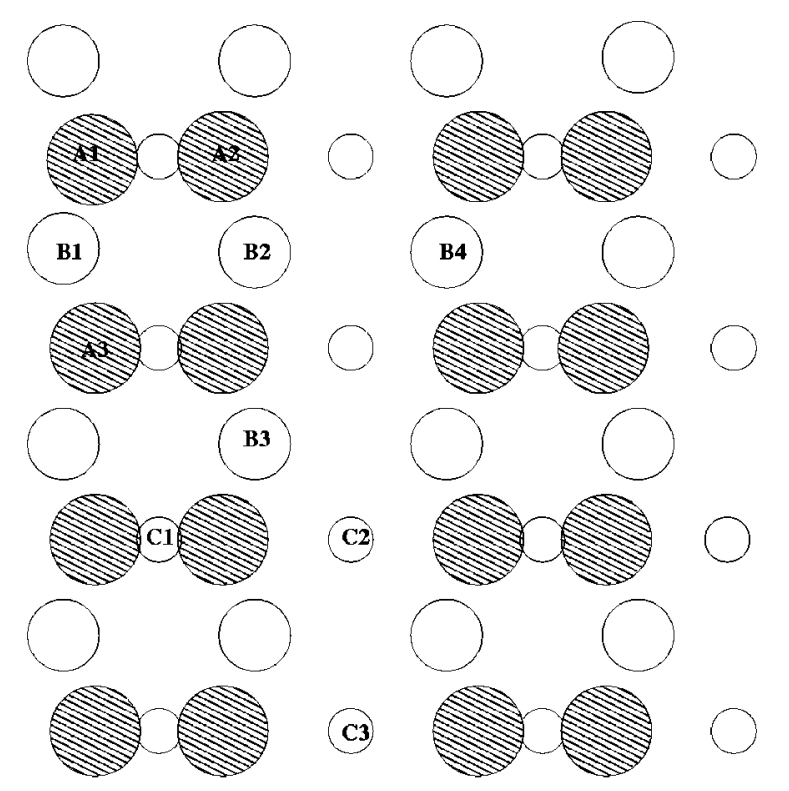

\section{1st Layer Si}

\section{2nd Layer Si}

\section{3rd Layer Si}

FIG. 2. Salient configurations of pairs of B impurities near the surface. The Si atoms in the first layer, which form dimers, are marked with large shaded circles, while those in the second and third layers are shown with successively smaller circles. Labels A1, $\mathrm{B} 1$, and $\mathrm{C} 1$ denote impurity positions. figurations near the surface were found to be repulsive by over $0.3 \mathrm{eV} /$ pair. In fact, the configuration with the impurities in one surface dimer is repulsive by about $0.4 \mathrm{eV} /$ pair.

The lowest energy configurations of the pairs were found in the second layer. There are three nearly degenerate orientations, denoted B1-B2, B2-B3, and B3-B4 in our notation, all with negligible binding energies with respect to isolated impurities (of the order of $0.01 \mathrm{eV}$ ). In the structures B1-B2 and B2-B3, the impurities are in a next-nearest-neighbor arrangement, placed directly underneath a surface dimer row and bonded to one surface $\mathrm{Si}$ dimer. In the third configuration, B3-B4, the impurities occupy positions at the opposite sides of a trench between dimer rows. Several other configurations in the second layer were considered and found to be repulsive by over $0.2 \mathrm{eV} /$ pair. Impurity pairs in the third or any deeper subsurface layer were found to be much higher in energy than the low-energy second layer configurations, by over $1.0 \mathrm{eV} /$ pair. In summary, the preferred modes of pairing of segregated B impurities place them in the second layer of the surface.

\section{SUBSURFACE ORDERING AT HIGH DOPING LEVELS}

When the doping level at the surface becomes a significant fraction of a surface monolayer, the impurities tend to form ordered structures. In the $(4 \times 4)$ supercell used in our calculations, there are sixteen inequivalent atoms per surface layer. A number of impurity ordering modes were investigated using 4, 8, 12, and 16 impurities per surface cell, which corresponds to $0.25,0.5,0.75$, and $1.0 \mathrm{ML}$. The total number of possible impurity configurations is very large. We used the intuition gained from calculating the energies of B pairs to select candidate low-energy structures. In the following, the energies of various ordered structures are presented with reference to the equilibrium energy of a second layer B pair, placed in the same $(4 \times 4)$ supercell.

For 0.25 ML doping, Fig. 3 shows schematic pictures of the studied configurations obtained by tiling subsurface B pairs. It should be noted that there are several ordered structures with substantial binding energies compared to isolated impurity pairs, ranging from -0.1 to $-0.3 \mathrm{eV} /$ pair. Overall, these results reveal certain striking qualitative trends: (a) The favorable modes of ordering generally involve zigzag alternation of clusters of the form B-B, Si-Si, B-Si parallel or perpendicular to the surface dimer rows; the only exception to this trend is the alternation of clusters of four B atoms underneath a surface $\mathrm{Si}$ dimer with similar clusters of four $\mathrm{Si}$ atoms. (b) Structures with chains of B impurities parallel or perpendicular to the surface dimer rows are unfavorable.

The configurations that we investigated at $0.5 \mathrm{ML}$ doping are also consistent with these trends. The results are depicted in Fig. 4. The lowest energy configuration, shown in Fig. 4(a), corresponds to zigzag ordering of a cluster of four second-layer B impurities bonded to one surface Si dimer, with an overall $c(4 \times 4)$ symmetry. Its energy is $-0.31 \mathrm{eV}$ per impurity pair, somewhat greater than that of the lowest energy configuration at $0.25 \mathrm{ML}$ ordering. Straightforward linear tilings of the clusters of four B atoms, shown in Figs. 4(b)-4(c) are significantly higher in energy. There are also large differences between the energies of tilings oriented parallel or perpendicular to the surface Si dimer rows. The two 

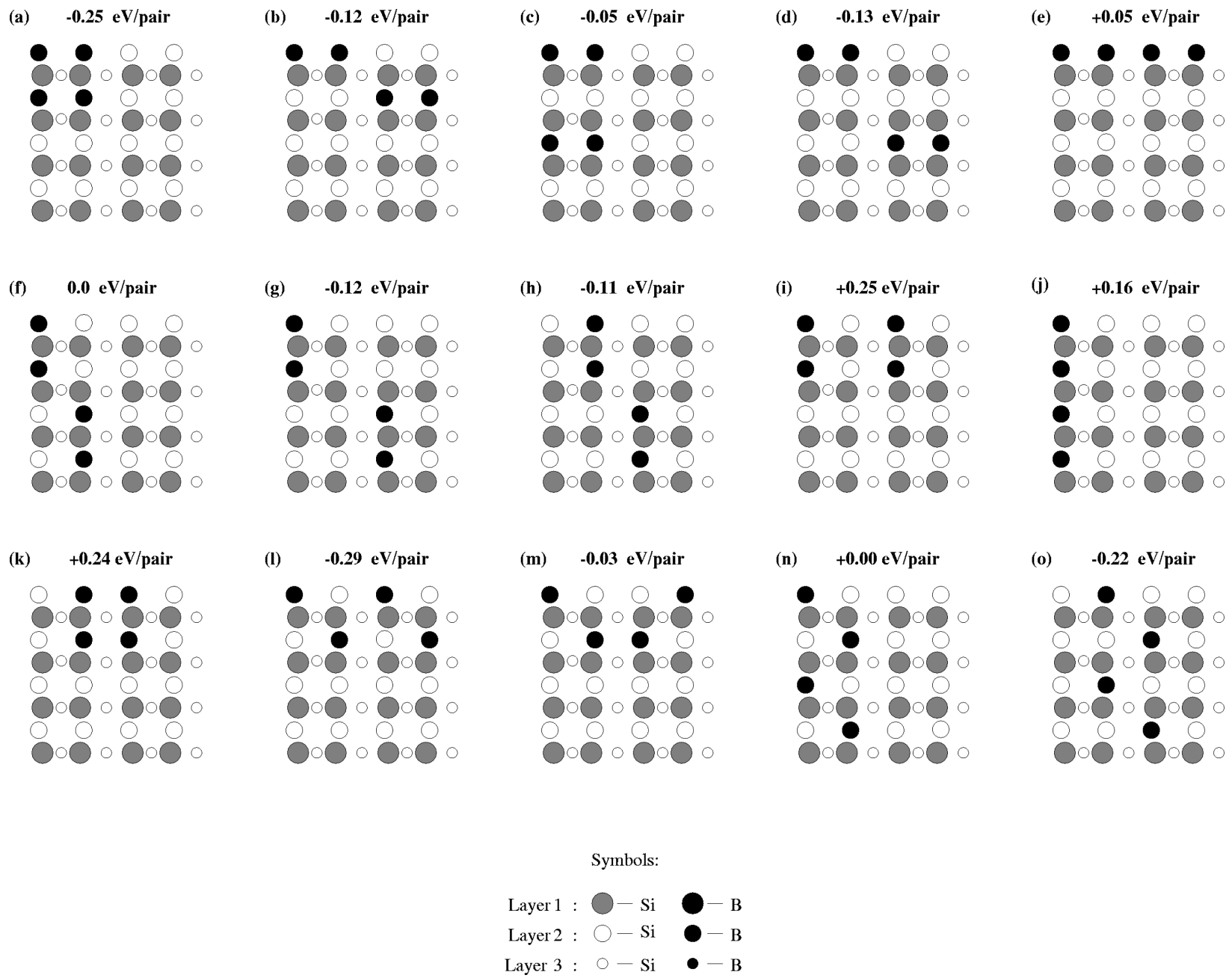

FIG. 3. Schematic structures and energies for $0.25 \mathrm{ML}$ surface doping. In this figure and the succeeding figures, the energies of the various ordered structures are presented with reference to the equilibrium energy of a second layer B pair, placed in the same $(4 \times 4)$ supercell. See also Fig. 1.

zigzag modes of ordering shown in Figs. 4(d)-4(e) are only a little higher in energy than the equilibrium configuration. Again, structures with linear chains of B impurities, shown in Figs. 4(g)-4(i), are all repulsive.

For $0.75 \mathrm{ML}$ ordering, several candidate structures were constructed, by adding B pairs to the structures shown in Fig. 4. The most favorable structure was found to have an energy of about $-0.02 \mathrm{eV} /$ pair, significantly lower than that at 0.5 ML doping. The formation of a full monolayer of B impurities was found to be highly unfavorable, in either the first or the second layer. The structure with $1 \mathrm{ML}$ of $\mathrm{B}$ atoms in the second layer is strongly repulsive, with energy of $+0.18 \mathrm{eV} /$ pair, while a monolayer of $\mathrm{B}$ impurities in the surface layer, has an energy of about $+0.56 \mathrm{eV} /$ pair.

\section{IMPURITY CLUSTERING IN THE ADLAYER}

To round off the discussion of impurity segregation and ordering, we report the results of a study of impurity configurations in the adlayer. The motivation for this derives from observations that other group III impurities (Al, Ga, and In) prefer ordered addimer configurations on this surface. ${ }^{16}$ Furthermore, models of boron ordering in the adlayer have been proposed by other investigators. $6,9,10$
We explored a number of adatom and addimer configurations of boron. Isolated adatoms were found to bind as addimers. Several configurations of boron addimers on the Si(100) surface are shown schematically in Fig. 5, along with their energies, referenced with respect to the subsurface boron pair B1-B2 and using the calculated chemical potential of bulk Si to compare structures with different numbers of $\mathrm{Si}$ atoms. The lowest-energy structure shown in Fig. 5(a) is higher in energy than the subsurface B pair by over $0.5 \mathrm{eV} /$ pair. Tiling the addimer configurations, as shown in Fig. 3, to form $(2 \times 2)$ structures that saturate all surface dangling bonds (as is preferred ${ }^{16}$ for $\mathrm{Al}, \mathrm{Ga}$, and In) yielded even more repulsive structures, with energies greater than +0.7 $\mathrm{eV} /$ pair. Therefore, the adsorbed configurations of boron are much higher in energy than the subsurface structures described earlier.

\section{DISCUSSION}

The theoretical results described so far reveal that doping the $\mathrm{Si}(100)$ surface with boron gives rise to a fascinating variety of two-dimensional modes of ordering. The impurities are drawn towards the surface both for the purpose of strain relief and to saturate surface dangling bonds. The fa- 
(a)

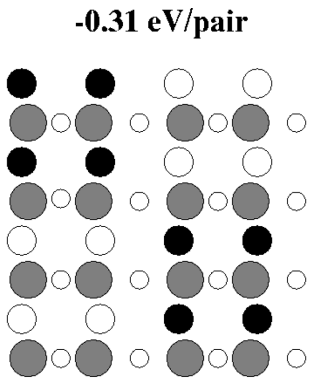

(d)

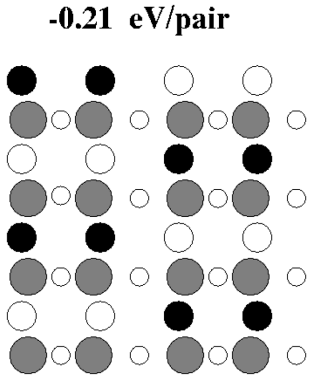

(g)

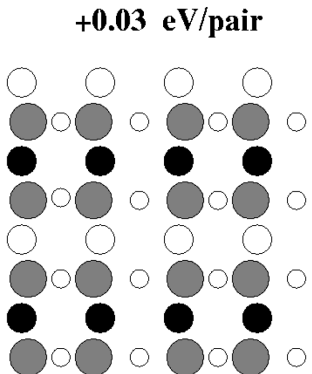

(b)

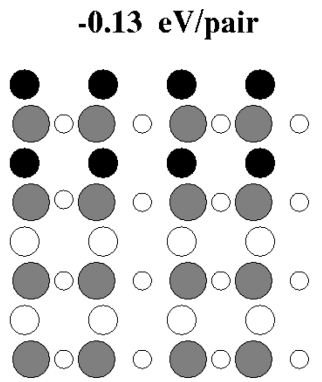

(e)

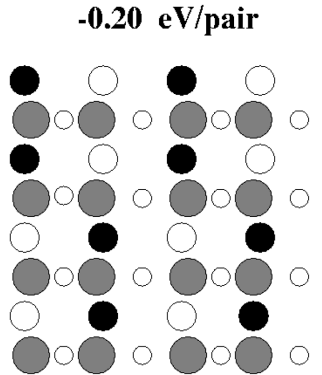

(h)

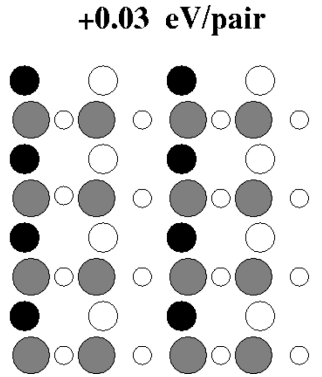

(c)

$+0.15 \mathrm{eV} /$ pair

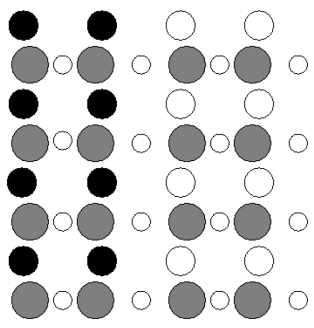

(f)

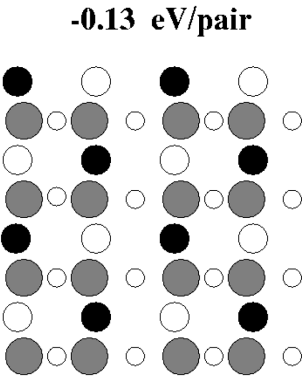

(i)

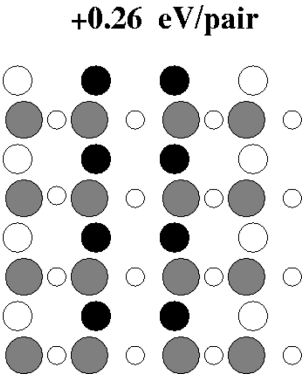

FIG. 4. Schematic structures and energies for 0.5 ML surface doping. The symbols for denoting Si and B atoms are the same as in Fig. 3. The energy reference is the same as in Fig. 3.

vored modes of ordering form by tiling subsurface clusters of two and four boron impurities in different periodic arrangements. The binding energies of these structures, with reference to isolated subsurface pairs, increases initially as a function of doping, reaches a maximum at $0.5 \mathrm{ML}$ doping and then decreases until, at 1.0 ML coverage, a highly repulsive structure forms.

Therefore, the critical doping level of 0.5 ML separates two qualitatively different regimes of boron aggregation. Below this doping level subsurface B pairs are able to arrange themselves in zigzag patterns that are attractive with respect to isolated pairs. However, at higher doping levels this is impossible. The incorporation of $\mathrm{B}$ above the level of 0.5 ML is also likely to be unfavorable kinetically, as the energies needed to add additional subsurface boron pairs to the structures shown in Fig. 4 are high. In practical terms, the $\mathrm{Si}(100)$ surface is "self-limited" to a boron doping level of $0.5 \mathrm{ML}^{3}$

\section{COMPARISON WITH EXPERIMENT}

The above results offer a qualitative explanation of a variety of known properties of the heavily B-doped $\mathrm{Si}(100)$ surface, while predicting the existence of a number of inter- esting low-energy structures that are yet to be identified. Indeed, in their pioneering studies, Headrick et al. ${ }^{3}$ did find that the $0.5 \mathrm{ML}$ doping level was a critical point in that the impurities readily formed ordered structures below this doping level, while above it they tended to disorder. The existence of several low-energy phases, nearly degenerate with the equilibrium configuration at half monolayer doping, is likely to be one reason why long-range ordering of any one phase is not observed, and only a $(2 \times 1)$ periodicity is observed as an average over a variety of domain structures. ${ }^{3}$ Indeed, STM investigations ${ }^{7}$ show that the size of an ordered domain is typically only $15-25 \AA$, well below the coherence length required for detection by LEED.

As we had noted in the discussions above, an important feature unifying all the impurity configurations are the large relaxations of $\mathrm{Si}$ atoms neighboring the B impurities. Such large relaxations should be observed experimentally, especially via STM, which is highly sensitive to the depth and spatial arrangement of surface atoms. In Fig. 6 we show the relaxed structure of the lowest configuration at $0.5 \mathrm{ML}$ doping, corresponding to Fig. 4(a), along with a simulated STM image of the occupied states within $0.5 \mathrm{eV}$ of the valenceband edge, generated using the Tersoff-Hamann approach. ${ }^{26}$ The results were found not to be particularly sensitive to the 
(a)

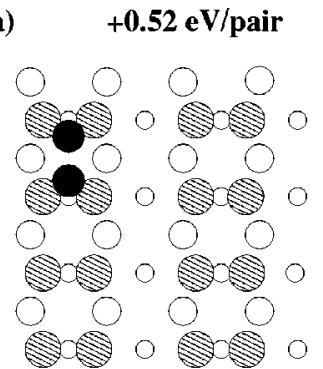

(b)

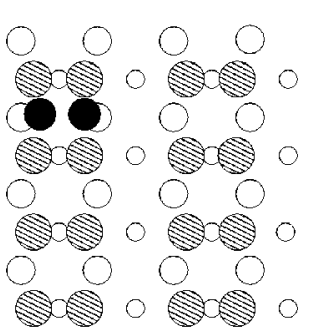

(c)

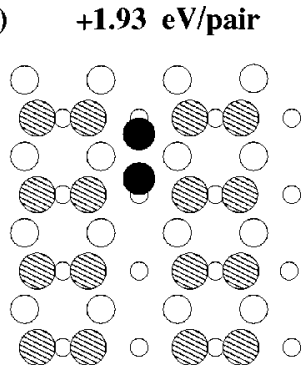

(d)

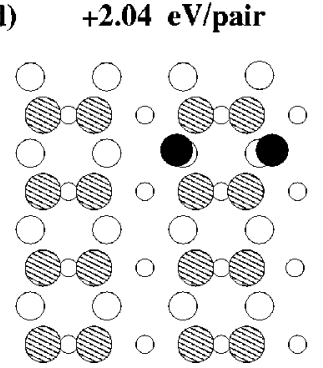

(e)

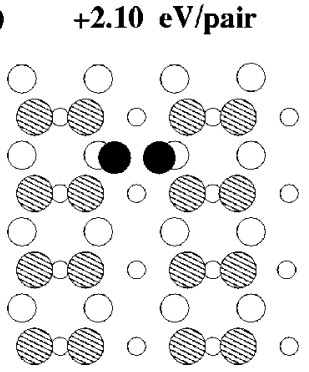

\section{1st Layer Si}

2nd Layer Sì

3rd Layer Si

Adsorbed B

FIG. 5. Various addimer configurations with energies quoted with reference to isolated equilibrium B pairs, as described in the caption to Fig. 3.

energy range of states included in the construction of the simulated image.

Figs. 6(a)-6(b) show different views of the surface atoms in the relaxed structure, and demonstrates that the surface $\mathrm{Si}$ dimers bonded to the subsurface boron pairs are greatly lowered with respect to those that have no boron neighbors. None of the $\mathrm{Si}$ dimers bonded to subsurface $\mathrm{B}$ atoms are visible in a simulated STM image [Fig. 6(c)], unlike those that have no subsurface B neighbors. In fact, the latter Si dimers are tilted, indicating the presence of dangling bonds at the surface. This result is consistent with the observations of Cao, Yang, a nd Pianetta, ${ }^{6}$ namely that the heavily doped surface has an appreciable density of dangling bonds, though reduced from that of the clean surface.

We propose that this $c(4 \times 4)$ reconstructed structure, shown schematically in Fig. 4(a), is identical to that observed by Wang, Hamers, and Kaxiras ${ }^{7}$ on heavily B-doped $\mathrm{Si}(100)$, and designated by them as the $\alpha$ phase. The subsurface arrangement of boron atoms is exactly as they had proposed. However, according to our results, the features that they interpreted as being ordered arrays of surface divacancies correspond to the lowered surface Si dimers above the subsurface B pairs. In fact, the calculations show that the energy cost to remove these B bonded surface Si dimers is about 1.5 $\mathrm{eV}$ per dimer. This is much higher than the formation energy of $0.2 \mathrm{eV}$ of a surface divacancy on the clean surface. ${ }^{17}$ Furthermore, our results show that this reconstruction does not need hydrogen for stabilization, as had been suggested recently. ${ }^{8}$ However, we are unable to explain the nature of the $\beta$ and $\gamma$ reconstructions observed by Wang, Hamers, and Kaxiras $^{7}$

We now address the experimental observations at low doping levels. Zhang et al. ${ }^{8}$ used STM to investigate boron accumulation at B-doped $\mathrm{Si}(100)$ via high-temperature annealing. They observed boron induced features, appearing as protrusions in the unoccupied state image, which replace two adjacent $\mathrm{Si}$ dimers of a surface dimer row. They proposed a model where these features correspond to pairs of B atoms in the third layer of the surface, together with two surface divacancies in the surface layer. More recently, Komeda and Nishioka ${ }^{9}$ reported STM images that revealed similar protrusions, in addition to other uncorrelated features that appear to be depleted dimers. The protrusions were assigned to a configuration where two B adatoms are placed on opposite sides of a surface dimer row, bonded to two adjacent Si dimers. The depleted dimers were interpreted to correspond to surface B dimers, substituting for Si dimers.

Our results presented above show that isolated and paired impurities in the third layer have high energies, over 1.0 $\mathrm{eV} /$ pair, compared to the second layer of the surface. Introduction of surface divacancies near such structures raises their energies even further. Moreover, in STM simulations, we do not find surface dimer vacancies to be imaged as protrusions, even in the presence of subsurface boron atoms. The paired impurity-adatom configuration proposed by Komeda and Nishioka, ${ }^{9}$ shown in Fig. 5(d), is very high in energy compared to the equilibrium configuration of a subsurface boron pair or a boron addimer. In Fig. 7, we show the simulated STM image for this structure using unoccupied states in an energy range around $2.0 \mathrm{eV}$ above the conduction-band edge. It is seen that the paired B adatoms are not imaged as being significantly protruded above the surface Si dimers. Therefore, none of the structures proposed to explain the protrusions seen in the unoccupied states image correspond to low-energy configurations, nor are likely to explain the protrusions seen in STM experiments. In Fig. 8 , we show the simulated STM image for the boron addimer structure shown schematically in Fig. 5(a), again using unoccupied states in an energy range around $2.0 \mathrm{eV}$ above the conduction-band edge. Indeed, this structure, which is $0.5 \mathrm{eV}$ 
(a)

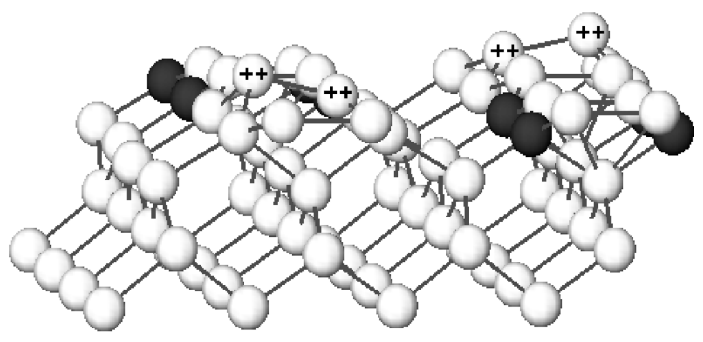

(b)

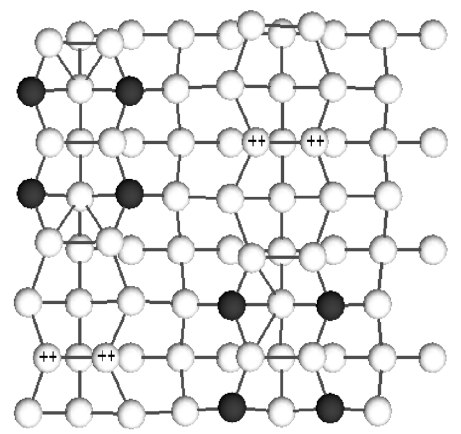

(c)

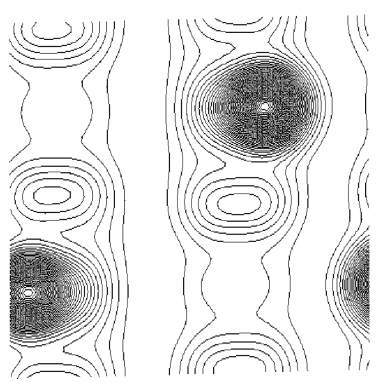

FIG. 6. Atomic configuration and simulated STM image for the equilibrium structure with $0.5 \mathrm{ML}$ B. The two surface Si dimers which have no B neighbors are marked with " ++ " in (a) and (b). Only these are imaged in (c). (a) Side view; (b) top view; (c) simulated STM image.

higher in energy than the equilibrium subsurface B pair, is imaged as a protrusion which replaces a pair of adjacent surface Si dimers. However, it is still possible that other low-energy impurity configurations are also in accord with the published STM data.

The depleted surface dimers are more easily accounted for in terms of low-energy structures. Surface Si-B or B-B dimers are greatly lowered compared to the average height of the Si-Si dimers which do not have B neighbors. Subsurface B impurities, on the other hand, are bonded to two adjacent surface $\mathrm{Si}$ dimers in a dimer row and tend to lower both tremendously. Such lowered dimers are not visible in our simulated STM images. As a concrete example we present in Fig. 9 a simulated STM image, obtained from occupied states around $2.0 \mathrm{eV}$ below the valence-band edge, for the subsurface boron pair labeled B1-B2. In this structure, the two surface Si dimers bonded to the subsurface B impurities are drawn into the surface, by almost $0.5 \AA$, and are not imaged at all. The image obtained using unoccupied states is (a)

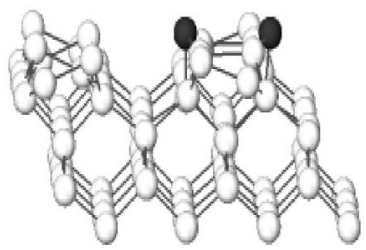

(b)

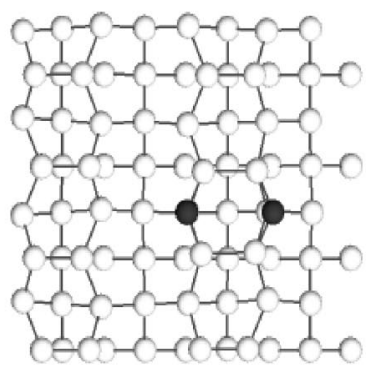

(c)

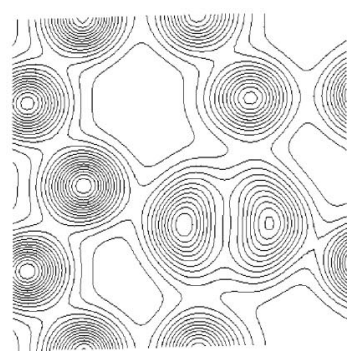

FIG. 7. Atomic structure and simulated STM image for the adlayer B pair shown in Fig. 5(d). (a) Side view; (b) top view; (c) simulated STM image.

qualitatively similar. Interestingly, the relaxations of the $\mathrm{Si}$ atoms appear to be very strongly localized around the impurities. The surface Si dimers that are not bonded to the impurity atoms cannot be distinguished in their appearance from those of the clean surface. In summary, while our calculations shed light on the structures observed at light and high doping levels, some STM observations remain unexplained.

\section{COMPARISON WITH EARLIER THEORY}

In the last three years several groups have reported totalenergy calculations of various structural arrangements of boron impurities at the $\mathrm{Si}(100)$ surface. Radny and Smith, ${ }^{10}$ Chang and Stott, ${ }^{11}$ and Fritsch et al. ${ }^{12}$ used $a b$ initio HartreeFock methods, density functional theory and a self-consistent multicenter local-orbital formalism, respectively. The investigated structures were restricted to those considered by the authors to be consistent with the published STM data and focussed on a $0.5 \mathrm{ML}$ boron coverage.

The lowest energy structure found by Radny and Smith consists of an array of B addimers with the orientation shown in Fig. 5(b) and having $(2 \times 2)$ periodicity. Chang and Stott studied a number of structures containing $\mathrm{Si}$ and $\mathrm{B}$ addimers. They concluded that none of the structures they had investigated, nor those proposed by Wang, Hamers, and Kaxiras $^{7}$ could satisfactorily explain the salient STM features. Fritsch et al. ${ }^{12}$ focused on identification of the struc- 
(a)

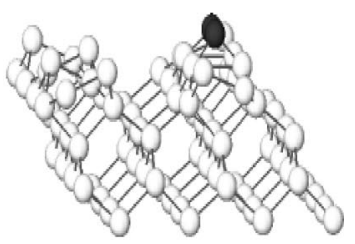

(b)

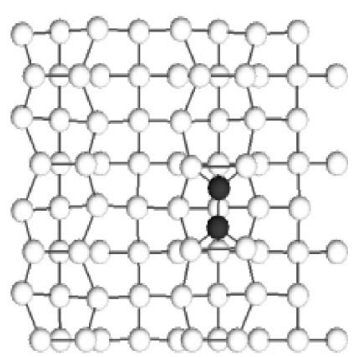

(c)

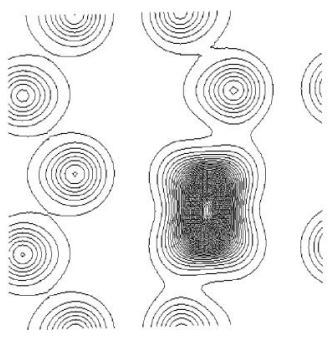

FIG. 8. Atomic structure and simulated STM image for the B addimer shown in Fig. 5(a). (a) Side view; (b) top view; (c) simulated STM image.

tural subunits that form the $c(4 \times 4)$ reconstructions reported by Wang, Hamers, and Kaxiras ${ }^{7}$ The lowest energy $c(4$ $\times 4)$ structure that they found has B impurities in the first and the third layers, forming a "rotated dimer model."

In this paper, our goal has been to obtain a systematic understanding of the preferred modes of ordering of B impurities, as a function of surface doping. We found that all heavily doped structures with B impurities in the adlayer, the first or the third layer of the surface are energetically unfavorable with respect to ordered structures in the second layer of the surface. However, we have not exhausted all possible arrangements of boron at the surface and thus cannot exclude the possibility of other low-energy configurations apart from those that we have investigated.

\section{SUMMARY AND CONCLUSIONS}

To summarize our results, the segregation and ordering of boron impurities at high-concentration levels near the $\mathrm{Si}(100)$ surface is due to a complex interplay between chemical and strain effects. Like the other acceptors in group III ( $\mathrm{Al}, \mathrm{Ga}, \mathrm{In})$, boron is favored to segregate to the surface, in order to saturate surface dangling bonds and to relieve the strain arising from its large atomic size mismatch with host $\mathrm{Si}$ atoms. However, unlike other group III acceptors, it prefers substitutional configurations in the first and second layers of the surface. ${ }^{16,17}$ With increasing doping, aggregation of boron impurities in the second layer of the surface be- (a)

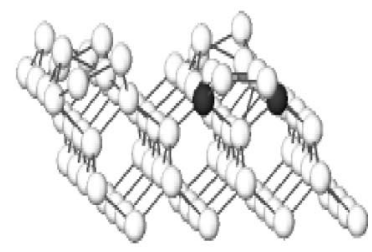

(b)

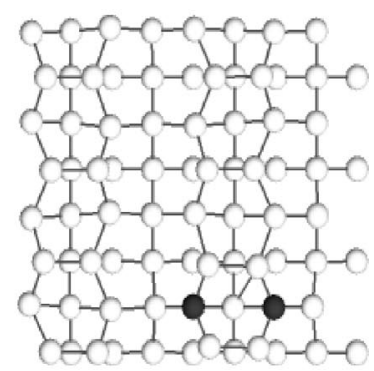

(c)

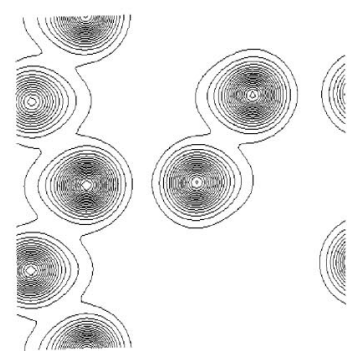

FIG. 9. Atomic structure and simulated STM image for one low-energy configuration of a subsurface boron pair. The two surface Si dimers bonded to the subsurface boron impurities are not imaged. (a) Side view; (b) top view; (c) simulated STM image.

comes more and more favorable, up to a critical level corresponding to $0.5 \mathrm{ML}$ of the impurities. Low-energy modes of ordering correspond to zigzag patterns of subsurface pairs. Above the critical doping level, the binding energies of ordered impurity configurations decrease sharply, and they become highly repulsive for a complete surface or subsurface impurity monolayer.

A number of experimental observations may be explained on the basis of our results. The equilibrium structure found at 0.5 ML doping has $c(4 \times 4)$ symmetry. However, both at low and high doping levels, several distinct modes of ordering are found to be essentially degenerate. This favors the formation of domain structures over long-range ordering of any one structure. Therefore, even at $0.5 \mathrm{ML}$ coverage only $(2 \times 1)$ LEED patterns are observed, ${ }^{3,6}$ while domains with $c(4 \times 4)$ and $(4 \times 4)$ periodicities are observed in STM. ${ }^{7}$ If some of the metastable ordered structures can be stabilized, there would be a potential for engineering complex impurity patterns at the nanoscale.

One common feature of all the surfaces with subsurface boron atoms is the pronounced flattening and lowering of surface Si dimers with B neighbors, of up to $40-50 \%$ of the interlayer spacing. As indicated in Fig. 6 for the equilibrium structure at $0.5 \mathrm{ML}$ doping, the lowered $\mathrm{Si}$ dimers are not visible in simulated STM images. However, the removal of such boron-bonded Si dimers to create surface divacancies is energetically very expensive, much more so than on the 
clean $\mathrm{Si}(100)$ surface. ${ }^{17}$ Therefore, the strain fields induced by heavy boron doping are not relieved by the creation of ordered patterns of surface Si divacancies. These results offer an alternative viewpoint concerning the dark features extensively observed on B-doped $\mathrm{Si}(100)$ in STM investigations. $^{7-9}$

In conclusion, boron impurities prefer to form twodimensional aggregates in the second layer of the $\mathrm{Si}(100)$ surface. The preferred modes of ordering are in complex registry with the $(2 \times 1)$ dimer row reconstruction, and may be constructed from different tilings of subsurface B pairs.
Both at high and low doping levels several distinct impurity configurations are degenerate or nearly degenerate in energy, indicating the possibility of forming complex domain structures with interesting structural and electronic properties. A surface doping level corresponding to $0.5 \mathrm{ML}$ of the impurities is found to be critical, in the sense that additional surface doping decreases the energy gain per B atom. Although a substantial number of possible impurity configurations near the $\mathrm{Si}(100)$ surface were considered, certain STM observations remain unexplained and will require further investigation.
${ }^{1}$ B. S. Meyerson, F. K. LeGoues, T. N. Nguyen, and D. L. Harame, Appl. Phys. Lett. 50, 113 (1987).

${ }^{2}$ H. Jorke and H. Kibbel, Appl. Phys. Lett. 57, 1763 (1990).

${ }^{3}$ R. L. Headrick, B. E. Weir, J. Berk, B. S. Freer, D. J. Eaglesham and L. C. Feldman, Phys. Rev. Lett. 65, 1128 (1990).

${ }^{4}$ B. E. Weir, R. L. Headrick, Q. Shen, L. C. Feldman, M. S. Hybertsen, M. Needels, M. Schluter, and T. R. Hart, Phys. Rev. B 46, 12861 (1992).

${ }^{5}$ C. P. Parry, R. A. Kubiak, S. M. Newstead, T. E. Whall, and H. C. Parker, J. Appl. Phys. 71, 118 (1992).

${ }^{6}$ R. Cao, X. Yang, and P. Pianetta, J. Vac. Sci. Technol. B 11, 1455 (1993).

${ }^{7}$ Y. Wang, R. J. Hamers, and E. Kaxiras, Phys. Rev. Lett. 74, 403 (1995); J. Vac. Sci. Technol. A 13, 1431 (1995).

${ }^{8}$ Z. Zhang, M. A. Kulakov, B. Bullemer, I. Eisele, and A. V. Zotov, J. Vac. Sci. Technol. B 14, 2684 (1996); Appl. Surf. Sci. 103, 443 (1996).

${ }^{9}$ T. Komeda and Y. Nishioka, Appl. Phys. Lett. 71, 2277 (1997).

${ }^{10}$ M. W. Radny and P. V. Smith, Vacuum 46, 419 (1995).

${ }^{11}$ J. Chang and M. J. Stott, Phys. Rev. B 53, 13700 (1996).

${ }^{12}$ J. Fritsch, J. B. Page, K. E. Schmidt, and G. B. Adams, Phys. Rev. B 57, 9745 (1998).

${ }^{13}$ R. I. G. Uhrberg, R. D. Bringans, R. Z. Bachrach, and J. E.
Northrup, Phys. Rev. Lett. 56, 520 (1986).

${ }^{14}$ J. Nogami, A. A. Baski, and C. F. Quate, Appl. Phys. Lett. 58, 475 (1991).

${ }^{15}$ Y. Wang, X. Chen, and R. J. Hamers, Phys. Rev. B 50, 4534 (1994).

${ }^{16}$ J. E. Northrup, M. C. Schabel, C. J. Karlsson, and R. I. G. Uhrberg, Phys. Rev. B 44, 13799 (1991).

${ }^{17}$ M. Ramamoorthy, E. L. Briggs, and J. Bernholc, Phys. Rev. Lett. 81, 1642 (1998).

${ }^{18}$ W. Kohn and L. J. Sham, Phys. Rev. 140, A1133 (1965).

${ }^{19}$ D. R. Hamann, M. Schluter, and C. Chiang, Phys. Rev. Lett. 43, 1494 (1979).

${ }^{20}$ E. L. Briggs, D. J. Sullivan, and J. Bernholc, Phys. Rev. B 52, R5471 (1995); 54, 14362 (1996).

${ }^{21}$ D. J. Chadi, Phys. Rev. Lett. 43, 43 (1979).

${ }^{22}$ R. J. Hamers, R. M. Tromp, and J. E. Demuth, Phys. Rev. Lett. 55, 1303 (1985); Phys. Rev. B 34, 5343 (1986).

${ }^{23}$ M. C. Payne, N. Roberts, R. J. Needs, M. Needels, and J. D. Joannopoulos, Surf. Sci. 211, 1 (1989).

${ }^{24}$ J. Tersoff, Phys. Rev. Lett. 74, 5080 (1995).

${ }^{25}$ P. C. Kelires and E. Kaxiras, Phys. Rev. Lett. 78, 3479 (1997).

${ }^{26}$ J. Tersoff and D. R. Hamann, Phys. Rev. Lett. 50, 1998 (1983); Phys. Rev. B 31, 805 (1985). 